Questions de communication

$24 \mid 2013$

Renouvellement des mises en scène télévisuelles de la politique

\title{
Parole d'experts, public profane : les mutations du discours politique à la télévision
}

From Insiders to Outsiders: The Advent of a New Political Television

Jeffrey P. Jones

Traducteur : Pierre Bouillon

\section{CpenEdition}

\section{Journals}

Édition électronique

URL : http://journals.openedition.org/questionsdecommunication/8671

DOI : 10.4000/questionsdecommunication.8671

ISSN : 2259-8901

\section{Éditeur}

Presses universitaires de Lorraine

\section{Édition imprimée}

Date de publication : 31 décembre 2013

Pagination : 97-123

ISBN : 978-2-8143-0182-5

ISSN : 1633-5961

Référence électronique

Jeffrey P. Jones, «Parole d'experts, public profane : les mutations du discours politique à la télévision », Questions de communication [En ligne], 24 | 2013, mis en ligne le 01 février 2016, consulté le 20 avril 2019. URL : http://journals.openedition.org/questionsdecommunication/8671 ; DOI :

10.4000/questionsdecommunication.8671 


\title{
PAROLE D'EXPERTS, PUBLIC PROFANE : LES MUTATIONS DU DISCOURS POLITIQUE À LA TÉLÉVISION
}

\begin{abstract}
Résumé. - Dans un premier temps, l'article décrit les évolutions du discours politique télévisuel en examinant le débat politique à la télévision depuis l'ère des grandes chaînes traditionnelles jusqu'à la première génération des chaînes câblées (donc jusqu'à la fin des années 80). Dans un deuxième temps, il étudie la manière dont une série de mutations et de changements survenus dans les sphères économique, politique, culturelle et technologique de la société américaine au début des années 90 a constitué un terreau propice à l'avènement de nouveaux programmes politiques. Enfin, la contribution analyse les changements du paysage télévisuel dus à la concurrence acharnée des chaînes câblées et leurs répercussions : prise de risques accrue, adoption de nouveaux formats, mise en place de nouvelles relations avec le public. Les programmateurs des chaînes câblées ont notamment proposé de nouveaux cadres du discours politique télévisé, d'abord sous l'impulsion d'animateurs aux accents populistes imitant le style radiophonique et ouvrant leur antenne à une parole politique profane, parfois même à des citoyens mécontents, sans parler d'autres animateurs, véritables tribuns ultraconservateurs. Ces nouveaux formats télévisuels n'étant pas économiquement rentables, ils ont été supplantés par deux nouvelles chaînes câblées d'information (Fox News et MSNBC) à l'origine d'une nouvelle approche du traitement de l'information caractérisée par l'abandon des traditionnelles émissions d'actualités télévisées au profit de débats marqués par l'idéologie.
\end{abstract}

Mots clés. - Télévision, politique, talk shows, expertise, divertissement. 
$\mathrm{D}$ e tout temps, la diffusion du discours politique à la télévision s'est fondée sur un principe essentiel : ceux qui prennent la parole sont censés posséder une connaissance incontestable d'experts sur le sujet débattu'. Pour les producteurs de télévision, cette expertise est la caractéristique inhérente à ceux qui ont la parole, qu'il s'agisse de personnalités politiques occupant le devant de la scène ou bien de leurs conseillers et stratèges, ou bien encore des journalistes et éditorialistes dont le métier est précisément d'analyser et commenter les faits et gestes des premiers. Une telle hypothèse s'appuie sur l'idée que le débat politique est essentiellement destiné à informer ou instruire, écartant, de fait, toute autre fonction de communication politique. Toutefois, ce dogme induit une série de conséquences logiques : les thèmes abordés, les questions à débattre et les acteurs politiques, tout ce qui constitue la structure même de la politique, sont à l'évidence le produit de cette expertise ; le public ne jure plus que par l'opinion des experts politiques et toute autre forme de discours politique perd sa légitimité dans la sphère publique qu'est la télévision.

À l'évidence, une telle conception, d'une part, des intervenants et, d'autre part, des publics découle d'une culture politique exigeant des citoyens qu'ils soient bien informés, une culture qui a érigé la pensée politique raisonnée en discours de référence ${ }^{2}$. Par ailleurs, elle est liée à une époque où sociologues, journalistes et même philosophes étaient plus en vue dans le dialogue politique national tenu dans la presse et les magazines de grande diffusion, une époque d'avant l'avènement de la télévision. Comme le rappellent certaines anecdotes du débat politique télévisé, cette vision des choses a son origine dans l'histoire des services d'information des grandes chaînes traditionnelles qui ont créé les talk shows et dans le fait que les journalistes se voyaient en arbitres des discussions et opinions politiques (Alterman, 1999 ; Hirsch, 1991).

Plus de 30 ans ont passé et ces hypothèses ont évolué. Bien que la parole des experts soit toujours dominante dans les programmes politiques des grandes chaînes traditionnelles et du câble, les années 90 ont vu l'émergence de nouveaux programmes et de nouvelles chaînes du câble proposant des approches clairement inédites du discours politique télévisuel autour de formats originaux $x^{4}$.

\footnotetext{
' Note de l'éditeur: le présent article est issu du chapitre : Jones J. P., 2005, « From Insiders to Outsiders :The Advent of New Political Television », in : Jones J. P., Entertaining Politics. New Political Television and Civic Culture, Oxford, Rowman and Littlefield Publishers (Tous droits réservés).

Cette traduction a été réalisée par Pierre Bouillon (université catholique de l'Ouest) et avec l'aimable autorisation de l'auteur et des ayants droit que Questions de communication et les coordinateurs du dossier remercient vivement.

2 Voir l'analyse de Michael Schudson (1998) sur les périodes de culture civique de l'histoire américaine et, entre autres archétypes, celui du citoyen informé, né des réformes de l'ère progressiste du début du $x x^{\mathrm{e}}$ siècle, modèle selon lequel la société américaine fonctionne encore aujourd'hui à bien des égards.

3 Eric Alterman (1999) propose une analyse intéressante de l'histoire de l'expertise politique à la télévision remontant aux écrits de Walter Lippmann dans la presse écrite. Voir aussi BernardTimberg (2002).

${ }^{4}$ Les taux d'audience recueillis par l'institut de sondage Nielsen (Nielsen Media Research) pour les talk shows du dimanche matin révèlent que plus de huit millions d'auditeurs suivent encore ce genre
} 
Ces changements ont été accompagnés d'un nouveau discours produit non plus seulement par des experts politiques, mais aussi par des personnalités éloignées de la logique et des opinions habituelles des élites politiques. Ces voix et ce programme d'un nouveau genre ont bousculé les présupposés sur le savoir des experts, les personnes habilitées à prendre la parole, les questions à aborder et ce qui pouvait être soumis à la critique ou non.

\section{Paroles d'experts à l'ère des grandes chaînes généralistes}

Dans l'histoire de la télévision, les débats politiques ${ }^{5}$ se sont généralement inspirés du journalisme, et plus spécifiquement des pratiques d'interview et de rédaction d'op-ed ${ }^{6}$. Les exemples les plus anciens sur les chaînes traditionnelles sont Meet the Press sur NBC (1947) et Face the Nation sur CBS (1954), émissions dans lesquelles des journalistes de presse et de radio interviewaient des membres du gouvernement et des personnalités du moment (Ball, 1998). À l'évidence, les intitulés de ces émissions montrent que les journalistes, par leur stratégie de questionnement, jouent pleinement leur rôle de porte-parole du grand public et de ses intérêts. Aujourd'hui, cette tradition se perpétue dans ces mêmes émissions et dans celles qui leur ont succédé, comme Nightline ou The NewsHour with Jim Lehrer. L'autre format classique de débat politique télévisé est la table ronde réunissant des journalistes. Cette table fit sont apparition en 1969 avec l'émission Agronsky and Company, animée par le journaliste de radio et de télévision Martin Agronsky diffusée sur la télévision publique. S'inspirant de la tradition journalistique de l'« op-ed », l'émission réunissait autour de Martin Agronsky quatre journalistes qui analysaient les événements de la semaine. Elle partait du principe que les journalistes étaient les observateurs indépendants les plus proches des événements survenant dans l'arène politique. Ils étaient donc à même d'émettre une opinion aussi avertie et impartiale que possible sur les faits. L'émission, qui allait plus tard devenir Inside Washington, a servi de modèle à un plusieurs autres comme Washington Week in Review (1967 sur PBS), The McLaughlin Group (I 982 sur PBS) ou The Capital Gang ( 1988 sur CNN).

d'émission. À titre d'exemple, le taux moyen d'audience des talk shows d'experts du dimanche matin diffusés dans les programmes télévisés de l'année 2006-2007 a été de 3 millions pour Meet the Press, 2,3 millions pour Face the Nation, 2 millions pour This Week et un million pour Fox News Sunday.

${ }^{5}$ L'analyse aborde la question du débat politique télévisé apparu en même temps que la première génération de diffusion de programmes sur le câble, à savoir sur CNN dans les années 80 . Bien qu'étant diffusée sur le câble, cette forme de débat politique était très semblable à celle proposée par la télévision publique et les grandes chaines, mais avec quelques modifications forçant l'aspect spectacle. Ce n'est que dans les années 90, avec l'apparition de nombreuses chaines câblées (ce que j'appelle la seconde génération des programmes sur le câble), que le discours politique d'expert s'est vu concurrencé par d'autres formes de débats et a pu se développer en ayant recours à des caractéristiques similaires à ces dernières.

${ }^{6}$ Note du traducteur : le terme « op-ed » désigne les articles d'opinion signés publiés sur la page opposée à l'éditorial. 
C'est en référence à ce type d'émissions que les critiques ont donné aux participants le sobriquet de «pundits », un mot d'ancien sanscrit signifiant « savant ». Mais, à mesure que le mot entrait dans l'usage courant de la langue anglaise, il fut employé à la fois pour désigner une personne dont l'avis fait autorité, mais aussi pour « railler ceux qui se permettent de critiquer sans cesse les politiques par le moyen d'observations n'ayant rien de secret et déjà largement présentes dans les médias » (Nimmo, Combs, 1992:6). Selon leurs critiques, de tels débats d'experts ne se contentent pas de jouer la mouche du coche mais sont par essence quelque peu dangereux; en effet, les journalistes qui y participent sont enclins à pérorer sur toutes sortes de sujets et d'événements qu'ils connaissent en réalité très peu. Si bien que leur expertise n'est pas celle du penseur mais bien plutôt du beau parleur. Comme le soulignent Dan Nimmo et James E. Combs (1992: 8) : « Ils contribuent aujourd'hui activement à mettre en place et en forme les opinions, à définir les grandes préoccupations du moment et à les évaluer, au point de transformer les États-Unis en "gouvernement des 'experts' [punditocracy]" : un pays où dominent les opinions diffusées par des journalistes célèbres omniprésents dans les médias ».

Le dernier type de talk show politique est en quelque sorte un assemblage des deux premiers. II met en scène un ou deux commentateurs qui discutent avec un invité plus qu'ils ne l'interviewent, créant ainsi un contexte dans lequel les opinions s'expriment librement, tout en se rapportant aux personnalités politiques du moment. L'émission pionnière, et à bien des égards emblématique de ce sous-genre, est Firing Line. Distribuée sous licence et proposée pour la première fois en 1966 par RKO, elle mettait en vedette William F. Buckley Jr ( 1989), un ultraconservateur, fondateur de la National Review. Des programmes similaires furent créés par la suite, comme Crossfire (en 1982 sur CNN) et, dans une certaine mesure, This Week with Davis Brinkley (en 198I sur ABC). Dans l'émission Firing Line, William F. Buckley prenait ce concept de débat politique télévisé au sérieux et avait recours à toutes sortes de figures de style (loyales ou parfaitement déloyales) pour avoir le dessus sur ses invités. Le producteur alla même jusqu'à imaginer le débat comme « une version intellectuelle de Friday night at the Fights $\rangle^{7}$ (Hirsh, 1989 : | 3). Le discours politique plus que brutal de William F. Buckley, volontiers parsemé d'injures, de menaces physiques, d'interruptions et de remarques humiliantes, a par la suite servi de modèle à bien des émissions politiques.

En fait, si la typologie des talk shows proposée jusqu'ici est fondée sur leurs caractéristiques structurelles et sur le casting des animateurs, une approche plus efficace serait peut-être de classer les débats politiques par catégories selon leurs tendances idéologiques et le style de discours qu'ils offrent. En suivant cette méthode, la progression logique va du formalisme pointilleux et du conservatisme libertarien d'après-guerre à la William F. Buckley jusqu'au style agressif et néoconservateur façon Ronald Reagan de John McLaughlin, en passant par celui bêtement hâbleur et

\footnotetext{
7 Matchs de boxe diffusés le vendredi soir à la télévision américaine (note du traducteur).
} 
farouchement réactionnaire de Bill O'Reilly (The O'Reilly Factor sur Fox News), le roi du discours politique belliqueux sur la télévision câblée. Cette autre classification permet également de constater un fort déclin de la qualité du discours politique depuis l'époque de Firing Line, auquel s'est ajouté le triomphe idéologique du conservatisme. William F. Buckley, dont la plupart des gens, et même ses pires ennemis, s'accordent à reconnaitre la grande intelligence, a engendré Bill O'Reilly qui a réussi, à lui seul, à prouver qu'un présentateur de talk show n'avait besoin d'aucune connaissance pour disserter sur n'importe quel sujet dans la plus parfaite ignorance, mais en attirant l'audience la plus importante pour un débat politique télévisé, tout en étant un auteur à succès. Son talk show n'est bien sûr qu'un exemple parmi les nombreuses illustrations du discours politique proposées à la grande loterie des émissions des chaînes câblées : Fox News, MSNBC, CNN et CNBC.

Bien que cet inventaire de la catégorie « discours politique d'experts » reste superficiel, les analyses détaillées (Hirsch, 1991 ; Alterman, 1999 ; Nimmo, Combs, 1992) conduisent à trois conclusions majeures sur la nature de ces émissions, leurs participants et le discours proposé. La première, et peut-être la plus importante, est que le point de vue indépendant et impartial que cette forme journalistique prétend avoir à l'égard de la politique n'est en fait ni l'un, ni l'autre. Plus précisément, bien que la plupart des experts tiennent des chroniques dans de grands journaux ou des hebdomadaires d'actualité, leur participation à des débats politiques télévisés a clairement montré qu'ils étaient étroitement liés aux sphères du pouvoir. En effet, plusieurs de ces « experts » éminents (ou leur épouse) ont travaillé pour divers gouvernements ${ }^{8}$. Ils participent tous activement à la vie politique, utilisent souvent un cadre épistémologique que l'on pourra qualifier de washingtonien et contribuent à l'orthodoxie et à la circulation des messages politiques émanant de la capitale fédérale. Plus accablant encore, ils concourent grandement à la propagation de rumeurs, aux règlements de compte, aux luttes de pouvoirs intra-bureaucratiques, caractéristiques de la vie politique à Washington?. Alice Mundy (1996), reporter à MediaWeek souligne le rôle majeur des débats politiques du dimanche matin dans les intrigues politiques de Washington : « Ces talk shows ne sont pas de simples divertissements, pas plus qu'ils ne se contentent de commenter l'actualité, les hommes et les femmes politiques s'en servent pour faire des annonces, créer des remous, envoyer des signaux à leurs alliés ou à leurs opposants. Ils s'en servent pour tester leurs propres stratégies d'emballage et de marketing de leurs idées ». En résumé, ces « experts » ne sont pas des commentateurs du système, eux et leurs talk shows constituent le système.

\footnotetext{
${ }^{8}$ Pat Buchanan écrivait les discours de Richard Nixon et a également travaillé pour l'administration George Bush. John McLaughlin était un ami personnel de Ronald Reagan et sa femme a été nommée secrétaire d'État au travail par ce dernier. Chris Matthews a travaillé pour Jimmy Carter. George Will était un ami proche de R. et Nancy Reagan, et sa femme a travaillé pour l'administration R. Reagan à la Maison Blanche et dans l'équipe de campagne de Bob Dole lors de sa candidature à la présidentielle de 1996.

${ }^{9}$ Le cas Robert Novak, expert de la télévision et chroniqueur qui a révélé être un agent de la CIA sur l'ordre de certains fonctionnaires « anonymes » de l'administration G.W. Bush en 2003, est sans doute l'exemple le plus récent et le plus flagrant des luttes de pouvoir au sein de la vie politique à Washington.
} 
La deuxième conclusion, en lien avec la première, est que les experts de la télévision ne sont pas de simples journalistes, mais des célébrités à part entière. Comme l'expert Robert Novak le fait remarquer : « Aujourd'hui, lorsqu'on me reconnaît, c'est en tant que célébrité de la télévision, et non pas comme commentateur travaillant à la télévision! » (Hirsh, 1991). À ce titre, ils fréquentent la bonne société des beaux quartiers de Washington et entretiennent des relations personnelles avec les politiques, et même avec plus d'un président. Non seulement ils reçoivent des salaires beaucoup plus élevés que leurs homologues ne travaillant pas pour la télévision, mais ils profitent de leur célébrité pour réclamer des honoraires faramineux en tant que conférenciers (Nimmo, Combs, 1992 : 43-44). En résumé, ils sont la face visible de l'opinion politique et spécifiquement attentifs à leur image, comme le sont toutes les célébrités, en restant dans le giron du star system qui les a créés ${ }^{10}$.

Le dernier, et crucial, enseignement qui peut être retiré de cette télévision de prétendus experts, est que la réalité tord le cou à la théorie selon laquelle ceux qui ont le plus de connaissances en matière de politique offriraient le discours politique le plus pertinent. La télévision de ces « experts » a clairement démontré que ces mises en scène publiques sont le plus souvent purement et simplement du spectacle. Les envolées rhétoriques de William F. Buckley ont abouti à de véritables spectacles de cirque généralisés, et l'émission McLaughlin Group en est sans doute l'exemple le plus flagrant. Indépendamment des émissions où ils interviennent, la plupart ont tiré les leçons des recettes de la « bonne » télévision. Un comportement calme, réfléchi, introspectif et prêt au compromis ne fait pas partie des ingrédients. Le public lui-même admet qu'il y a un côté « spectacle » dans les talk shows, mais les railleries des participants et la connivence qui existe entre eux finissent par lasser le public, sauf ceux qui sont toujours demandeurs d'informations d'initiés et, en outre, sont capables de supporter l'ambiance agressive de ces spectacles.

Pour résumer, la forme dominante du discours politique à la télévision n'est plus conforme aux idéaux journalistiques d'objectivité, de pondération, de pensée rationnelle dont ils sont censés être issus. S'y sont substitués des programmes mettant en vedette des célébrités intimement liées au pouvoir en place, participant à un système de réflexion politique étriqué et proposant un spectacle verbeux sans grand intérêt, sauf pour les connaisseurs des choses de l'État ou les accros à la politique. Bien que d'aucuns aient fait observer que ces émissions servaient plus à graisser les rouages de l'establishment qu'à informer et instruire l'électorat, ils représentent l'unique option ou presque dans le paysage audiovisuel en termes de discours politique. À leur tour, les spectateurs et électeurs ont reçu le message des médias : « La politique, tu l'aimes ou tu

10 Comme le prédit Alan Hirsch (199| : |82-183), parce que le succès engendre l'imitation, la plupart des commentateurs se trouvent aujourd'hui sur le chemin de la célébrité et ne tiendront probablement pas compte de l'avertissement du célèbre expert Jack Germond : « Si la célébrité vous empêche de faire votre travail correctement, alors c'est un vrai problème et cela implique d'être extrêmement prudent ». 
l'ignores ». Et comme il se doit, le taux extrêmement bas de participation aux présidentielles et aux législatives de 1988 et 1990 a démontré que les gens se désintéressaient massivement de la politique.

En conséquence, alors qu'une série de changements intervenait dans le contexte économique et politique des années 80 et du début des années 90 , les producteurs de télévision ont pris acte des failles du système pour proposer de nouvelles formules de débats politiques télévisés susceptibles d'intéresser le public. Toute accusation remettant en cause la légitimité de ces nouveaux formats de débats politiques télévisés en raison de leur présentateurs vedettes, du droit de parole donné à des non-experts ou encore de leur ressemblance avec des émissions de divertissement semblait contestable en raison de la nature même de la télévision de ces soi-disant « experts ». Toutefois, avant d'analyser ce genre d'émission, nous devons comprendre les changements politiques, technologiques, culturels et économiques survenus au sein de l'industrie des médias qui furent à l'origine de ces nouveaux formats d'émissions.

\section{Des changements de contexte}

Le facteur essentiel qui a modelé la politique et la culture politique des années 80 fut l'élection de Ronald Reagan et sa popularité. Cette célébrité d'Hollywood avait su conserver tout le crédit lui permettant de jouer efficacement du décalage que lui autorisait son statut d'outsider, c'est-à-dire de candidat n'appartenant pas au sérail politique, et de lancer sa litanie aux accents populistes selon laquelle l'état n'est pas une des sources de nos problèmes, mais bel et bien LE problème. La popularité de Ronald Reagan s'est construite en majeure partie sur sa prise de position anti-gouvernement - ce même gouvernement étant présenté comme un handicap pour la société américaine - et non sur des politiques ou des programmes bénéfiques pour la grande majorité des Américains qui le soutenaient" ${ }^{\prime}$. Lors de l'élection présidentielle de 1988, les candidats républicain et démocrate Pat Robertson et Jesse Jackson ont tenté de reprendre le flambeau du populisme de Ronald Reagan en menant des campagnes d'outsiders (Hertzke, 1993). Leurs campagnes électorales se soldèrent par un échec, mais la rhétorique populiste à laquelle ils avaient eu recours allait refaire surface deux années plus tard à l'occasion des élections législatives de mi-mandat : ce malaise d'essence populiste allait, de fait, alimenter plusieurs campagnes sur le thème du « débarrassons-nous de ces voyous » (Oreskes, 1990 ; Dillin, 1990). Deux années plus tard, lors des élections présidentielles, ce malaise populiste fut incarné par l'outsider Ross Perot

\footnotetext{
" Selon Robert Dallek (1999:8, |4,24) : « Les déclarations de Reagan sur n'importe quel sujet, que ce soit l'avortement ou l'aide sociale, étaient plus symboliques que fondamentales [et prouvaient] son extraordinaire maîtrise des symboles nationaux qui trouvaient un écho si retentissant chez des millions d'Américains ». Ses « objectifs pour la nation devaient satisfaire des besoins à la fois psychologiques et matériels ».
} 
qui mit en avant une approche du gouvernement dite de « sens commun » lors de réunions publiques locales. II s'agissait de savoir précisément ce que « le peuple » attendait d'un gouvernement ${ }^{2}$. Ross Perot fut également le premier à participer à des shows télévisés populaires non politiques et à les utiliser comme principal moyen de communication avec le public. Sa candidature elle-même découlait de son apparition dans l'émission Larry King Live où il déclara aux téléspectateurs que si le peuple américain voulait de lui comme candidat, il était prêt à financer lui-même sa campagne (King, Stencel, 1993). À mesure que la campagne présidentielle avançait, tous les candidats participèrent à des talk shows « populistes » similaires, pour dialoguer en direct avec « le peuple » et éviter les conférences de presse et les questions généralement agressives des journalistes (Dowd, 1992). Deux ans plus tard, de nombreux citoyens américains avaient rejoint le camp républicain qui, entre-temps, avait pris le train populiste en marche en promettant des garanties législatives infaillibles sous forme de « contrats » écrits passés avec le public ${ }^{13}$ (Mansfield, 1994).

Un des éléments de langage de cette vague populiste fut le « sens commun » que citoyens et politiques adoptèrent comme s'il se fût agi du Saint Graal de la crise de la légitimité, la panacée censée ramener les responsables politiques et les technocrates, tous aussi ingérables les uns que les autres, sur la voie de la raison, de la transparence et de l'efficacité (Dionne, 1991 ; Lipset, Schneider, 1987 ; Pharr, Putnam, 2000). Le vice-président Al Gore (1995) s'efforça de convaincre les électeurs de la pertinence de son rapport promettant une meilleure efficacité du gouvernement en reprenant et en singeant une publicité pour une bière : « Le gouvernement du Sens Commun : il travaille mieux tout en coûtant moins cher ». I| fut donc nécessaire d'expliciter clairement cette rhétorique du sens commun qui en vint à constituer le dixième commandement du « contrat avec l'Amérique » du Parti républicain et donna lieu à une loi présentée au Congrès sous le nom de «The Common Sense Legal Reform Act of 1995 » (« Loi de réforme de 1995 sur le sens commun ; The New York Times, 1995 ; Klein, 1995).

Une certaine perméabilité entre la sphère politique et les médias vit le jour à cette période. Le traditionnel chassé-croisé entre les personnels du service public et de l'industrie médiatique en vint à concerner des personnes beaucoup plus en vue. Des responsables politiques qui avaient bénéficié de toute l'attention des médias lorsqu'ils occupaient un poste de fonctionnaire d'État ou de candidat d'un parti politique se voyaient maintenant promus au rang de célébrités en quittant leurs fonctions pour rejoindre de grands groupes de médias désireux d'exploiter leurs noms et leurs

12 Pour une analyse du « populiste » R. Perot, voir Dennis Westlind (1996) et Linda Shulte-Sasse (1993:91-119).

13 Pour une évaluation plus générale des années 90 perçues comme une époque politique « populiste », voir Sean Wilentz (1995), Paul Piccone et Gary Ulmen (1995). Pour un commentaire sur les accents populistes de la décennie et leur décalage avec la vraie définition du populisme, voir Molly lvins (1996). 
célébrités. Oliver North ${ }^{14}$, Mario Cuomo ${ }^{15}$, Ross Perot ${ }^{16}$, Jesse Jackson ${ }^{17}$, Jerry Brown ${ }^{18}$, Susan Molinari ${ }^{19}$, George Stephanopoulos ${ }^{20}$, David Gergen ${ }^{21}$ et Pat Buchanan ${ }^{22}$ entre autres exemples, se sont tous recyclés, d'une manière ou d'une autre, dans les médias. Cependant, cette mue « des responsables politiques en célébrités médiatiques » ne faisait que refléter les difficultés croissantes de la société américaine à s'identifier aux partis politiques et son besoin de plus en plus pressant de pouvoir choisir les responsables politiques parmi des individus qu'elle aurait « appris à connaître » grâce à leur exposition médiatique (Marshall, 1997). Au fur et à mesure que la déception de la société américaine envers le gouvernement augmentait, les responsables politiques prenaient leurs distances par rapport aux structures politiques traditionnelles et jouaient du décalage pour se positionner en outsiders politiques, libre de toute contrainte mise à part celle d'obtenir un mandat du « peuple ».

14 Note de l'éditeur (NDÉ) : né en 1943, O. North est un ancien lieutenant-colonel du corps des marines. En 1994, il est le candidat républicain au poste de sénateur de Virginie et, depuis 200 I, il anime l'émission War Stories with Oliver North sur Fox News. En outre, il est l'auteur de plusieurs ouvrages.

15 NDÉ : né en 1932, avocat et membre du parti démocrate, M. Cuomo est élu gouverneur de l'État de New York en 1982 et réélu à ce poste en 1986 et 1990.

16 NDÉ : né en 1930, R. Perot est un homme d'affaire milliardaire. En 1992, il se présente en tant que candidat indépendant à l'élection présidentielle des États-Unis d'Amérique. En 1995, il fonde le Parti réformateur et, en 1996, est de nouveau candidat à l'élection présidentielle mais, cette fois, au poste de vice-président. Depuis 2000, il signe et participe à plusieurs ouvrages.

17 NDÉ : né en 194I, J. Jackson est révérend, membre du Parti démocrate et militant pour les droits civiques, notamment ceux des noirs. En 1984 et 1988, il est candidat aux primaires de son parti pour les élections présidentielles.

18 NDÉ : né en 1938, J. Brown est avocat et membre du Parti démocrate. II occupe les postes de secrétaire d'État de Californie (1970-1975), maire d'Oakland (1998-2006), procureur général de Californie et gouverneur de Californie (1975-1983; 201 I-).

19 NDÉ : née en 1958, S. Molinari est membre du Parti républicain et élue à la Chambre des représentants des États-Unis de 1990 à 1997. De 1997 à 1998, elle co-anime l'émission CBs This Morning sur CBS, puis plus tard, The Flipside (Channel 4).

${ }^{20}$ NDÉ : né en 196I, G. Stephanopoulos est conseiller politique. II travaille sur les campagnes présidentielles démocrates de Michael Dukakis en 1988 et de Bill Clinton en 1992. Durant la présidence de B. Clinton, il a occupé plusieurs postes au sein de son administration dont celui de conseiller principal en politique et stratégie. Ensuite, G. Stephanopoulos rejoint ABC News comme analyste politique et correspondant pour l'émission politique du dimanche matin This Week. Depuis 2002, il présente cette émission.

${ }^{21}$ NDÉ : né en 1942, D. Gergen rejoint l'équipe en charge des discours du président des États-Unis d'Amérique Richard Nixon (avec notamment Pat Buchanan) en 197I. En 1975, il est nommé directeur de la communication du président Gerald Ford puis, en 1980, conseiller pour la campagne présidentielle de George W. H. Bush. En 1993, il revient à la Maison blanche comme conseiller du président Bill Clinton puis du secrétaire d'État Warren Christopher.

22 NDÉ : né en 1938, P. Buchanan est diplômé en journalisme par l'université de Columbia. Il commence sa carrière journalistique au St. Louis Globe-Democrat avant de devenir conseiller politique de Richard Nixon, puis de Gerald Ford. De 1978 à 1984, il co-présente Buchanan-Braden Progam, intervient souvent pour des chroniques sur NBC et collabore avec la chaîne CNN comme chroniqueur régulier dans The McLaughlin Group, Crossfire et The Capital Gang. De 1985 à 1987 il est directeur de la communication de la Maison blanche sous la présidence de Ronald Reagan puis retourne sur CNN. En 2000, il change de chaîne pour MSNBC et anime Buchanan and Press jusqu'en 2003 avant de devenir analyste politique de la chaîne. En 2002, il co-fonde le magazine The American Conservative. 
En faisant l'inventaire des changements survenus dans la sphère politique pendant les années 80 et au début des années 90, on observe l'imbrication des mondes des people et du personnel politique, l'utilisation du « sens commun » comme élément de langage dans le discours et la pensée politique, et, simultanément, un regain de populisme antipolitique au sein d'une société trouvant le milieu politique de plus en plus détestable. La population s'est donc orientée vers les politiciens qui participaient à des talk shows télévisés parce que ce type d'émission échappait au discours politique sclérosé qu'elle avait pris l'habitude d'entendre. Les téléspectateurs se sont alors rendus compte qu'ils pouvaient eux aussi poser des questions aux candidats et que les réponses étaient beaucoup plus sensées et accessibles que le langage formaté utilisé par les communicants en d'autres lieux ${ }^{23}$. Dans le domaine technologique, l'inquiétude politique s'est conjuguée avec les attentes sociales et le potentiel d'innovation, le tout débouchant sur une certaine aspiration pour le populisme. Les années 90 ont vu les innovations des années 80 se développer grâce à la révolution du micro-ordinateur et de la télévision câblée. L'internet devenait une réalité commerciale, sociale et politique, notamment pour les citoyens des classes moyennes et aisées de l'époque. L'espoir d'un changement politique orchestré par les médias vit le jour dans un contexte de progrès technologique illustré par 500 chaînes câblées, des réunions publiques locales virtuelles, une communication possible à travers tout le village planétaire, des informations politiques facilement disponibles grâce à des débats présidentiels axés sur les citoyens, la participation des candidats à des talk shows que les auditeurs pouvaient appeler en direct, l'accès aux courriels des membres du Congrès et, enfin, la possibilité de rencontrer des personnes partageant des idées semblables aux siennes dans le cyberespace ${ }^{24}$. Les limites de l'espace et du temps semblaient avoir été repoussées et le fossé entre le discours des élites et celui des masses comblé. Les problèmes dus à des responsables politiques bornés ou à une bureaucratie tatillonne allaient disparaître à mesure que les gens auraient accès aux nouvelles technologies de communication et ces derniers pourraient donc participer aux décisions régissant leurs vies (ou, tout du moins, faire des choix mieux adaptés dans les menus informatiques proposés) ${ }^{25}$.

23 Lors des élections présidentielles de 1992, Thomas E. Patterson (1993) a comparé dix questions posées par des citoyens américains aux candidats et dix questions posées par des journalistes lors de débats de campagne ou de conférences de presse. L'une de ses conclusions en fut que la presse utilisait un langage totalement étranger aux citoyens et éloigné de leurs préoccupations.

24 Par exemple, John T. Caldwell (1995: 257) cite un ancien conseiller de la FFC (organisme fédéral de prévisions) et de la Maison blanche, également membre de la National Association : « L'offre pléthorique des chaînes câblées destinées à un créneau particulier du marché permettra de répondre aux besoins spécifiques de tel ou tel groupe culturel de notre société multiculturelle. En plus d'offrir des "possibilités de responsabilisation" aux minorités, ces réseaux câblés aux spécificités culturelles devront posséder des équipes dirigeantes réactives aux besoins de leur public cible ».

25 L'ouvrage d'Howard Rheingold (199I) est un travail très représentatif de cette vision utopiste. Pour une analyse critique des illusions engendrées par les technologies de communication, voir Theodore Roszak (1994). Les divisions entre les partisans de l'utopie et ceux de la contre-utopie devinrent si profondes à la fin de la décennie qu'un groupe de « modérés » a finalement créé ce 
Dans le domaine culturel, les citoyens ont conduit des batailles idéologiques dans le cadre de ce qui est souvent appelé des « guerres culturelles » (Gitlin, 1995). Dans les grandes lignes, le terme fait référence aux perpétuels désaccords entre les libéraux et les conservateurs à propos de sujets tels l'orientation sexuelle, l'appartenance raciale, l'égalité d'accès physique, les représentations médiatiques, la religion, la moralité publique et les relations hommes/femmes. Ces batailles ont été livrées au nom de valeurs et de comportements culturels (par exemple, les médias, le langage, le « style de vie », le monde universitaire, la religion) tout autant par des institutions sociales que dans le strict cadre de la vie politique. Pourtant, les terrains d'affrontement sont relativement fluctuants, au point que les conflits culturels peuvent se conduire dans un lieu de parole publique et politique (règlements judiciaires, procédures de destitution, etc. $)^{26}$ et les conflits politiques l'être depuis une tribune culturelle, par exemple un débat télévisé. Le terme « politiquement correct » fut employé par les conservateurs et les modérés pour condamner sur un ton moqueur les tentatives de la gauche et des progressistes pour faire évoluer ce qui était considéré dans la société comme des agissements dangereux, stéréotypés ou idéologiquement orientés. Selon ces critiques, le politiquement correct imposait certains types de comportements et, dans une société américaine fortement individualiste, des voix se sont souvent élevées publiquement, notamment lors de talk shows télévisés, pour condamner ces tentatives.

De même, dans le domaine de la culture populaire, les soaps, ces feuilletons à l'eau de rose de l'après-midi, ont cédé la place à des talk shows à thèmes produits sous licence. Ces émissions doivent leurs premiers succès à des animateurs masculins, comme Phil Donahue et Geraldo Rivera dans les années 80, mais c'est la popularité d'Oprah Winfrey, suivie de toute une génération de femmes comme Jenny Jones, Sally Jessy Raphael et Ricki Lake, qui a déclenché l'incroyable essor de ces talk shows de l'après midi au début et au milieu des années $90^{27}$. Objets de nombreux travaux de recherche universitaire, en général, ces talk shows traite des sujets touchant au domaine de l'intime, comme l'anorexie, la grossesse chez les adolescentes, l'inceste, l'homosexualité... et font intervenir tout autant des experts que des non-spécialistes dont les échanges sont orchestrés par un animateur. Au cours de

qu'il a appelé « le technoréalisme », un manifeste ancré dans la « réalité » qui devrait tous nous faire garder les pieds sur terre. Voir Andrew Shapiro (1998).

26 Pour Frank Rich, chroniqueur au New York Times, la procédure d'« impeachment » et le procès du président Bill Clinton sont un exemple de guerre culturelle. Dans le New York Times (19/12/98, AI5), il souligne que « les fractures culturelles de l'époque sont les mêmes qu'il y a trente ans, et potentiellement aussi explosives ; autrefois, les boucs émissaires de l'idéologie de droite étaient les hippies à cheveux longs, objecteurs de conscience, aux mœurs sexuelles dépravées (une caricature de la gauche, même à l'époque) et aujourd'hui c'est Bill Clinton, dont la politique opportuniste faites de concessions est en fait plus proche de celle du Parti républicain traditionnel que de la gauche des années 60, mais qui est néanmoins devenu l'emblème de ce que la droite hait le plus dans la révolution sexuelle et sociale des années 60 et 70 ».

27 Pour un échantillon représentatif des recherches universitaires conduites sur les talk shows à thèmes de l'après-midi, voir Wayne Munson (1995), Jane M. Shattuc (1997), Bernard Timberg (2002) et Andrew Tolson (1991). 
l'émission, les téléspectateurs sont invités à participer à la discussion et c'est sur ce point que les chercheurs ont constaté la capacité des non-spécialistes à remettre en cause la parole des experts professionnels (Livingstone, Lunt, 1994 ; Carpignano et al., 1993). Ces émissions ont depuis lors évolué vers ce que l’on appelle la « télé poubelle », où les invités viennent faire des révélations sur leurs déviances sexuelles ou leurs petites fautes personnelles, ou bien se retrouvent face à des membres de leur entourage sous l'œil des caméras afin d'offrir un spectacle insolite. Nombreux sont ceux qui s'accordent à dire que le roi et la reine de la télévision poubelle sont Jerry Springer et Ricki Lake ${ }^{28}$.

S'agissant de la dimension économique de l'industrie des médias, les producteurs se sont rendu compte que la « sagesse populaire » était une valeur sûre qu'ils pouvaient vendre comme un produit de consommation. Le format « débat radiophonique » fut une aubaine pour les radios locales AM dans tout le pays, grâce aux participants et aux auditeurs qui, par un discours politique populiste, contribuèrent à relancer un secteur qui battait de l'aile. L'animateur radiophonique Rush Limbaugh fut pionnier dans ce domaine, et son succès entraîna dans son sillage une foule de disciples à travers tout le pays, comme par exemple Ken Hamblin - «The Black Avenger » (« Le vengeur noir ») -, G. Gordon Liddy et Sean Hannity ${ }^{29}$. En prenant modèle sur le débat radiophonique tout en exploitant les nouveaux outils interactifs offerts par le boom de l'internet, les promoteurs de la télévision par câble ont alors créé des chaînes de débats et développé des stratégies de programmation visant à cristalliser un malaise aux accents populistes. Pour ce faire, ils donnèrent la parole au sens commun de l'Américain moyen et eurent recours aux technologies interactives pour mieux se rapprocher des téléspectateurs. CNN mit en place une émission quotidienne d'une heure, TalkBack Live (1994). Sur MSNBC, la première émission du genre fut America's Talking (1994). Multi-Media/Gannett créa le Talk Channel (1994) qui fut rebaptisé NewsTalk Television en 1995. C-SPAN proposa sa tribune libre du matin Washington Journal. Quant aux activistes du Parti républicain, ils créèrent le National Empowerment Television (1993) rebaptisé America's Voice en 1998, une chaîne qui s'affichait clairement comme « populiste 》. À peu près à la même époque, deux humoristes se firent remarquer dans des talk shows qui proposaient un nouveau et inhabituel genre de discours politique tout à la fois sérieux et divertissant : le Denis Miller Live, qui fit son apparition sur HBO en 1994 , et Politically Incorrect lancé en 1993 sur la chaîne humoristique Comedy Central, qui fut cédé quatre en plus tard à une grande chaîne traditionnelle.

Pour résumer, dès le début des années 90 existait un contexte dans lequel la logique et la pensée populistes ont pu être largement véhiculées pour traiter les préoccupations politiques. Dans ce contexte, le « sens commun » a servi de

${ }^{28}$ Doug Mclntyre, écrivain et invité de l'émission Politically Incorrect, a surnommé ces shows « des combats de coqs humains ». Voir Joshua Gamson (1998), Laura Grindstaff (2002) et Kevin Glynn (2000).

29 Pour des récits sur le succès des débats radiophoniques et les personnalités qui les ont portés, voir Peter Laufer (1995) et Howard Kurtz (1996). Pour une analyse de l'influence supposée des débats radiophoniques sur la politique, voir David C. Barker (2002). 
solution fourre-tout à tous les problèmes complexes, et la célébrité politique fut le critère d'identification du public, le tout débouchant sur un nouveau style de politique susceptible de proposer des solutions plus séduisantes. Dans cet environnement social, les technologies de la communication ont fait renaître l'espoir et l'optimisme et permis de vaincre la communication à sens unique de technocrates et de dirigeants lointains, en donnant aux gens les moyens de mieux se faire entendre, de mieux comprendre et de mieux choisir. Nous parlons d'un environnement culturel dans lequel les rivalités politiques s'expriment de plus en plus souvent dans des tribunes culturelles tels les talk shows. Simultanément, il s'agit d'un environnement économique dans lequel l'industrie médiatique se livre à une concurrence acharnée pour créer de nouveaux programmes et de nouvelles chaînes au prix le plus faible possible, tout en restant innovants et proches des goûts du public. II est essentiel de noter à quel point ces processus sont liés entre eux : le désenchantement pour la politique produit des réactions optimistes à la technologie et aux nouveaux médias ; les déceptions engendrées par le gouvernement trouvent une échappatoire dans la culture et notamment dans la valeur cardinale de la culture populaire qu'est la célébrité ; les guerres culturelles deviennent des guerres politiques (et vice versa) et constituent donc un contenu attractif pour des programmes médiatiques ayant le conflit comme fil conducteur. Ainsi la convergence technologique crée-t-elle des opportunités d'exploitation politique et économique, la concurrence économique engendre de nouveaux formats de programmes politiques faisant appel à la technologie.

Pour bien comprendre le style d'émissions politiques qui allaient voir le jour dans ce contexte, il est important d'examiner en détail la réaction spécifique de l'industrie télévisuelle devant la concurrence croissante des médias câblés dont l'offre se faisait toujours plus riche. En réalité, il serait plus utile de se demander quelles mesures ont été prises par les producteurs (notamment pour les chaînes câblées déjà existantes ou nouvellement arrivées sur le marché) non seulement pour paraître plus attrayantes auprès du public, mais aussi, du fait de cette attractivité, pour établir une relation inédite avec ce même public?

\section{Style télévisuel, participation du public et discours de profanes}

Dans la période qui vit la fin du monopole des chaînes traditionnelles (fin des années 80 et début des années 90), deux événements se produisirent en rapport avec notre sujet. D'une part, l'industrie de la télévision changea le style des programmes pour attirer de nouveaux publics par des moyens inédits et novateurs et, d'autre part, on constata simultanément le succès d'émissions de tribune libre distribuées sous licence et programmées l'après-midi, et leur influence sur l'évolution des idées sur des sujets comme l'autorité, le droit à la parole, l'accès à l'information et la possibilité d'apporter son témoignage sur les enjeux publics à la télévision. C'est sur 
le fondement de ces deux évolutions majeures que la nouvelle télévision politique s'est créée, accompagnée d'un nouveau genre de programmes permettant à des humoristes ainsi qu'à un public non expert d'exprimer leurs opinions de profanes.

Le premier de ces changements a été provoqué par la concurrence accrue à laquelle les programmateurs du câble se sont livrés envers l'oligopole des grandes chaînes traditionnelles. En effet, le câble a offert aux consommateurs un très vaste choix de chaînes parfaitement ciblées et donc bien adaptées à leurs goûts (sports, musique, informations, etc.). Pour les chaînes câblées déjà existantes ou pour celles nouvellement créées, l'enjeu était de proposer des contenus suffisamment intéressants et attractifs pour inciter les téléspectateurs à abandonner les chaînes historiques et les empêcher de se tourner vers d'autres offres concurrentes sur le câble. Les chaînes traditionnelles se voyaient obligées de mettre en avant une « image de marque » spécifique. En 1993, la course effrénée à l'audience conduisit l'un de leurs dirigeants à déclarer : « La routine ne va pas suffire. Nous devons trouver de nouvelles méthodes pour relancer nos activités si nous voulons survivre à la prochaine décennie » (Caldwell, 1995 : 292). Outre cette concurrence acharnée, l'industrie télévisuelle était confrontée à des bouleversements touchant la production, comme l'arrivée de nouvelles technologies audiovisuelles et le bouleversement des coûts de production. II fallait donc en passer par une nouvelle esthétique en termes de présentation et de nouveaux critères de séduction pour attirer le public.

John Thorton Caldwell livre sans doute l'analyse la plus complète et la plus instructive des réactions stratégiques de l'industrie télévisuelle. Les moyens auxquels les grandes chaînes traditionnelles eurent recours dans cette lutte pour leur survie impliquaient, selon lui, la mise en place d'un plan ambitieux d'innovation et d'évolution du style. Le nouveau look qui en est ressorti est ce qu'il appelle la « télévisualité » (« televisiuality »), un choix esthétique poussant à l'excès de style. « La télévision a abandonné son cadre traditionnel de référence fondé avant tout sur le langage et une transmission par les mots pour adopter un cadre et des usages nouveaux axés sur le visuel, avec une esthétique préoccupée à l'extrême par la recherche d'un style » (ibid. : 4). Ce style est devenu la question centrale, la ligne directrice de la télévision pour se différencier dans la course à l'audience. Cependant, l'excès de style n'est pas qu'un simple phénomène visuel. C'est un moyen d'imprimer un «look » qui donnera aux téléspectateurs l'impression de regarder des émissions originales.

La dynamique se cachant derrière ce nouveau besoin d'exhibitionnisme a été l'évolution de la relation entre les téléspectateurs et le produit télévisuel. « L'individuation et l'hétérogénéité sémiotique qui ressortent de cet excès télévisuel indiquent que ces émissions sont dès le départ conçues pour des publics bien ciblés, qui sont séduits par des revendications de différence et de distinction (ibid. : 25l) 》. Ces nouvelles règles concernent à la fois le public et l'industrie de la télévision, ainsi que les dispositifs qui fondent leur relation. Les téléspectateurs sont considérés par l'industrie comme des consommateurs pleins de jugeote et lucides sur eux-mêmes alors que ces dispositifs « exigent des positions plus affirmées de la part des téléspectateurs » (ibid. : 256). 
Concomitamment à cette reconfiguration, les émissions à thèmes de plus en plus nombreuses, distribuées sous licence et diffusées l'après-midi, ont connu une popularité grandissante et ont souvent été qualifiées de programmes ouverts à la participation en direct des téléspectateurs. Un large corpus de travaux de recherche universitaires a été consacré à l'analyse de ces programmes et à leur place dans la société. Sur deux points précis, les conclusions des chercheurs méritent toute notre attention. D'une part, ces émissions (en invitant les personnes présentes dans le studio à participer à la création même de ces programmes) ont permis au public de remettre en question ce qui constituait jusqu'ici « l'autorité » et « l'expertise » dans les débats télévisés sur des thèmes d'intérêt public, y compris de s'interroger sur la légitimité de celui qui entend parler et être entendu sur ces sujets. D'autre part, la manière dont ce type d'émissions a peu à peu brouillé les frontières entre les différents types d'émissions du genre talk show (dites de « divertissement » ou, au contraire, « sérieuses 》). Paulo Carpignano et ses collaborateurs (1993) estiment que les émissions ouvertes à la participation en direct du public « posent le problème de la distinction entre les experts et le public, le professionnel et le non-spécialiste ». D'après ces chercheurs, ces émissions « constituent un "espace de contestation" dans lequel de nouvelles pratiques de discours se mettent en place, différentes des modes traditionnels de représentation politique ou idéologique (ibid). À l'aune de ces échanges qui opposent souvent les « experts» aux « non-spécialistes », les auteurs soulignent le net rejet par le public présent dans le studio (et peut-être également des téléspectateurs) des propos tenus par les personnes faisant autorité : « Ce qui est exprimé ici est un refus non pas du savoir mais de l'expertise. Le talk show rejette l'arrogance d'un discours qui se définit par sa divergence d'avec le sens commun. Dans les débats, l'autorité de l'expert est remplacée par l'autorité d'une parole nourrie par l'expérience vécue » (ibid. : | | 6-| | 7 ).

De la même façon, dans leur étude sur les talk shows américains et britanniques, Sonia Livingstone et Peter Lunt (1994) arrivent à une conclusion analogue en reprenant la thèse de jürgen Habermas sur l'opposition entre le monde vécu et le système, à savoir la différence entre le savoir inhérent au vécu et le savoir spécialisé émanant de la logique professionnelle et institutionnelle du système. Ils font valoir que ces talk shows « adoptent un point de vue anti-élitiste qui s'inspirent clairement de traditions épistémologiques alternatives, proposent une réévaluation du monde vécu, refusent les reproches d'incompétence et d'ignorance faits à l'encontre de MonsieurTout-lemonde, contestent la déférence habituellement réservée aux experts en invoquant leur éloignement du monde vécu et leur appartenance à un système, et revendiquent les mérites des "gens ordinaires" » (ibid. : 102). Ils estiment aussi que cette évolution des schémas traditionnels est d'importance, ayant remarqué dans un grand nombre de médias britanniques l'amorce d'un mouvement « refusant de plus en plus les commentaires et les analyses critiques, au profit d'une parole redonnée aux gens ordinaires en lieu et place d'un réalisme social trop conscient de son œil critique $\gg^{30}$.

\footnotetext{
30 S. Livingstone et P. Lunt, (1994) prétendent que ce phénomène se remarque à la télévision
} britannique, dans les programmes documentaires, d'actualité et de théâtre. 
La seconde conclusion issue de ces recherches conduit à considérer les talk shows modernes comme un terrain de médiation spécifique, aux caractéristiques inter et multi-génériques, où la frontière entre le « sérieux », le « populaire » et le « divertissement » est de plus en plus floue (Tolston, |99| : 198). Wayne Munson ( 1993 ) déclare que la raison en est que le talk show a un format aléatoire et flexible et donc, par définition, hybride. L'auteur estime que le talk show « associe deux paradigmes de communication et, comme son nom l'indique, réunit et semble réconcilier deux discours différents, voire contradictoires. En effet, il associe la conversation et les échanges interpersonnels - la tradition orale prémoderne - au spectacle médiatisé né de la modernité » (ibid. : | 5). II offre un espace permettant au public d'établir des critères d'identification multiples et donne également aux producteurs la possibilité de « rafraîchir » le paysage télévisuel.AinsiWayne Munson explique-t-il que le talk show réunit, d'une part, le « professionnel » ou « l'expert » et, d'autre part, « l'amateur », un invité ou un quelconque participant, ce dernier étant présent en vertu de son expérience personnelle ou simplement en tant que membre de l'auditoire. II mélange habilement le traditionnel et le populaire avec le grand public, les échanges immédiats et interpersonnels avec les échanges médiatisés pour produire une dialectique efficace qui, simultanément, reflète et créé « un besoin insatiable de changement et d'innovation » d'une économie de l'image, et « d'évolution perpétuelle des règles et de changement de décor » ainsi que le souligne Andrew Ross (ibid. : 15). La conséquence de l'évolution de ces formats et autres réagencements est que le public de ces émissions est de plus en plus « fragmenté ». En écho à ce que dit John T. Caldwell à propos des producteurs de télévision qui se sont efforcés de créer de nouvelles relations avec des publics ciblés en les séduisant avec des arguments de distinction, Andrew Tolson (1991: 198) affirme « qu'il n'existe plus un public unique pour une télévision à vocation généraliste, ciblé par la publicité de masse, mais bien plutôt une diversité de publics appartenant à des chapelles et à des tribus, caractérisés par différentes sortes de "discernement averti" ».

En résumé, ces recherches font la lumière sur des particularités qui pourraient devenir les caractéristiques mêmes de la nouvelle télévision politique. Parmi ces particularités, signalons la construction multi-générique des programmations, l'introduction d'une « parole ordinaire », des points de vue divers exprimés pour interpeller la communauté des « experts », un style d'échanges informel et le recours à une logique de vulgarisation et de « sens commun » sur des questions habituellement abordées avec un langage et une connaissance de professionnel. Ces spécificités ont leur importance car elles offrent une approche qualitative différente du discours politique plus paternaliste proposé par la télévision des soidisant « experts ». Aux observations de John T. Caldwell sur l'exubérance stylistique de la télévision dans l'ère télévisuelle post-grandes chaînes nationales, s'ajoute le fait que les producteurs des nouvelles chaînes politiques aux accents populistes se sont reposés sur un discours politique d'insatisfaction pour imprimer leur style, proposer une présentation spécifique et tout en excès, le tout emballé dans l'engouement pour les nouvelles technologies de communication et leurs gimmicks. 
Qui plus est, les téléspectateurs se voyaient flattés par l'argument selon lequel leur voix ne comptait pas pour rien, et que l'Amérique n'attendait que leur opinion. Une nouvelle relation s'établissait autour de la participation du téléspectateur au discours télévisuel. Dans cette relation, le téléspectateur jouait le rôle valorisant du citoyen « engagé » qui contribuait à la création des programmes. Ainsi la recherche d'un style télévisuel, d'un look différent et nouveau, a-t-elle fait surgir de nouveaux modes de discours et de nouvelles formes de participation et de présentation du discours politique à la télévision, qui avaient jusqu'ici été ignorés ou négligés.

\section{Des programmes pour la voix du peuple}

Au milieu des années 90, est apparue sur le câble une poignée de chaînes offrant un choix éclectique d'émissions dont le discours politique était centré sur le public. NBC, Multi-Media/Gannett, CNN, C-SPAN, et la Free Congress Foundation ont alors consacré des émissions ou même des chaînes câblées entières à une parole politique de « profane » en s'inspirant du style des tribunes radiophoniques ${ }^{31}$. Avec ces émissions peu chères à produire, les groupes télévisuels surfaient non seulement sur la vague du succès des tribunes radiophoniques, mais également sur celle du discours populiste porté par un large mouvement antipolitique et antigouvernemental, tout en profitant de l'engouement pour les nouvelles technologies de communication. En réalité, les producteurs essayaient de répondre à l'insatisfaction des citoyens et des téléspectateurs envers la politique grâce au potentiel des nouvelles technologies de communication.Ainsi ont-ils eu l'opportunité de faire évoluer leur style et les contenus de leurs programmes à peu de frais, en bénéficiant de l'énorme avantage d'avoir un public réel sur les plateaux, ce qui n'était pas le cas des tribunes radiophoniques. Les analyses et les opinions politiques émises par les téléspectateurs pouvaient être exprimées pendant l'émission par courriel, fax, message vocal, appel téléphonique, forum de discussion, visioconférence ou encore par un système d'affichage électronique. Ces programmes, qui se voulaient élégants et de bon goût, étaient mis en scène dans un décor high-tech et donnaient à voir une batterie de gadgets dernier cri : télécopieurs, messages défilants, écrans d’ordinateurs, kiosques numériques dans les galeries et centres commerciaux, et autres écrans de visualisation pour faire entendre « la voix du peuple ».

Selon John T. Caldwell, la concurrence sur le marché du câble rendait obligatoire de tels marqueurs de style ainsi que la mise en place de nouveaux liens plus forts avec les téléspectateurs. Cette évolution des programmes câblés a modifié les relations temporelles et spatiales avec les téléspectateurs pour faire entendre « la voix du peuple ». En effet, les chaînes câblées ont encouragé les spectateurs à participer plus largement aux programmes avant, pendant et après leur diffusion, en prenant part aux discussions via des forums, des affichages électroniques, par courriel ou message

\footnotetext{
$\overline{31}$ Ce passage s'appuie sur une analyse complète de Jeffrey P. Jones (2003).
} 
vocal. Selon les dirigeants des réseaux câblés, le public était lassé d'un discours politique et social centré sur les élites, et désireux de voir apparaitre de nouveaux formats de débats télévisés lui permettant de témoigner et d'exprimer ses opinions.

Parmi les démarches allant dans ce sens, il faut noter l'initiative de NBC qui a su associer à bon escient la radiodiffusion et le câble en créant, le 4 juillet 1994, America's Talking, qui allait rejoindre MSNBC deux années plus tard, une nouvelle chaine dédiée à toutes les formes de talk shows. America's Talking (AT) permettait à NBC de faire évoluer son concept plutôt étriqué de talk show télévisé diffusé en prime time vers un format plus polyvalent. Avec à l'esprit l'énorme succès des tribunes radiophoniques, la chaîne a engagé Roger Ailes, ancien stratège du Parti républicain et producteur exécutif de la fameuse émission télévisée de Rush Limbaugh distribuée sous licence, pour piloter à la fois America's Talking et CNBC. Roger Ailes apportait à la nouvelle chaîne le dynamisme qu'il avait déjà testé avec Rush Limbaugh. II suffisait de regarder la liste des nouvelles émissions et le concept d'ensemble choisi pour la chaîne qui cherchait à reproduire la recette du succès de Rush Limbaugh, de son image d'« homme ordinaire », de sa hargne populiste et de sa colère contre la gauche.

Avec des titres comme Pork (sur le gaspillage et la corruption au gouvernement), Bugged! (présenté comme une «thérapie primale offerte au public par les autoroutes de l'information ») et Am I Nuts? (sur le stress de la vie quotidienne), Roger Ailes s'est efforcé de créer une chaîne servant d'exutoire à toutes les frustrations de la vie moderne dont étaient censés souffrir les téléspectateurs (Dempsey, 1994). II insistait également sur le fait que la chaîne « avait à cœur de représenter les vrais gens » (Brown, 1994). AT désirait être la première télévision à afficher sa différence en se fondant sur l'hypothèse selon laquelle le public ne se contentait plus de regarder passivement la télévision, mais désirait participer activement à l'élaboration des programmes. Douze des émissions d'AT avaient recours aux écrans d'affichage en ligne, sondages, courriels et autres forums de discussions (Williams, 1994). L'alliance de la technologie et d'une ligne politique populiste était destinée à séduire et à impliquer un certain public, sans attendre que ce dernier ne vienne tout seul à la chaîne, mais en s'efforçant, au contraire, de le créer de toutes pièces.

Le même élan populiste put être observé dans la programmation de la National Empowerment Television (NET), une petite chaîne du câble officiellement associée à la Free Congress Foundation, une organisation politique conservatrice fondée par Paul Weyrich, un activiste du Parti républicain. NET fut lancée le 6 décembre 1993 et se présentait comme une association à but non lucratif exonérée d'impôt. L'objectif premier de la chaîne n'était pas de faire des bénéfices, mais de peser sur la vie politique. Sa mission était simple : permettre au public de demander des comptes aux élites politiques (de gauche, cela va sans dire !). Selon ses dirigeants, cet objectif pourrait être atteint, d'une part, en élaborant des programmes qui se passeraient de l'élite médiatique, fournissant aux téléspectateurs des informations « exactes » et « impartiales »leur permettant de détecter les mensonges relayés par les grands médias et les élites politiques et, d'autre part, en mettant à la disposition des citoyens américains des moyens - des émissions interactives à lignes ouvertes - leur 
permettant de « rembarrer Washington » et donc de « pousser le gouvernement dans ses retranchements » (Wharton, 1993). Vice-président de la chaîne, Burton Pines déclara : «Nous avons un penchant pour le populisme. Les Américains se plaignent de Washington et du gouvernement et nous soutiendrons les Américains, pas Washington » (Moss, 1993). La chaîne voulut donner cette force et ce poids à son public (et s'assurer de sa fidélité), principalement à l'aide de ses programmes dont $80 \%$ avaient recours aux antennes ouvertes aux téléspectateurs. Toutefois, cette chaîne dont l'ascension fut concomitante de celle du populisme conservateur de Newt Gingrich et consorts, et de la « Révolution républicaine » de 1994, subit le reflux de ces mouvements et dut admettre qu'elle ne pourrait se maintenir économiquement. Elle se déclara en faillite en janvier 2000.

Le dernier exemple de cette tendance des chaînes du câble à donner la parole aux profanes fut CNN. Dans le souci d'augmenter son audience de l'après-midi, heure à laquelle aucune actualité brûlante ne méritait une importante couverture médiatique, la chaîne lança le 22 août 1994 son TalkBack Live. C'était un talk show d'une heure sur les affaires publiques, diffusé en plein milieu d'après-midi ( 15 heures, heure de la côte Est), qui se voulait le pendant idéaliste d'une assemblée publique traditionnelle. Lors du lancement, CNN présenta l'émission comme un forum national ouvert au dialogue public, un espace favorisant l'établissement de passerelles susceptibles de rapprocher les gens, un lieu où le public pourrait dialoguer avec les responsables politiques ayant le pouvoir de « changer les choses ». Alors que l'émission America's Talking avait recours à toute sorte de technologies interactives pour des raisons populistes et de style, TalkBack avait choisi une approche fondée sur un discours d'utopie démocratique - la technologie mise au service de la démocratie pour la revigorer - en donnant au public un accès au pouvoir et en rapprochant les Américains les uns des autres. « L'objectif est de recréer une réunion publique à l'ancienne, mais avec des moyens techniques modernes, pour établir des liens qui, à mon avis, nous font terriblement défaut, » put ainsi déclarerTeya Ryan, productrice exécutive de l'émission (Kloer, 1994). « Les gens ne s'intéressent pas simplement à ce que disent les experts, mais également à ce que disent leurs compatriotes » déclara-t-elle par ailleurs (Rice, 1994).

À l'instar de America's Talking, TalkBack utilisait de nombreuses technologies : liaisons téléphoniques, courriels, visioconférences et forums de discussion, pour donner la parole aux gens ordinaires au cours de l'émission. La toute première animatrice de l'émission, Susan Rook, déclara : «C'est comme le jeu vidéo Crossfire mais avec de vrais gens » (Sokolsky, 1994). Le plateau avait été conçu pour représenter la dimension interactive de l'émission et faire fusionner le monde des affaires, de la consommation et de la politique en un ensemble homogène. Installé dans le grand hall d'entrée des locaux de cNN au centre-ville d'Atlanta, le plateau pouvait recevoir I 50 personnes parmi lesquelles des touristes aussi bien que des autochtones, des habitants du quartier, des personnes venant y faire des courses ou y travailler. Outre le public assistant en direct à l'émission, les téléspectateurs avaient la possibilité d'intervenir et de faire passer des messages de chez eux, grâce à dix lignes téléphoniques, un télécopieur et un ordinateur 
installés sur une table au milieu du décor. Depuis les coulisses, les producteurs faisaient parvenir des témoignages et des questions de téléspectateurs par le truchement de vidéos réalisées hors studio et de commentaires postés en ligne, pendant que, sur le plateau, le télécopieur continuait à déverser des fax.

Mais à l'aube du XXle siècle, alors que l'élan populiste et l'engouement du pays pour la technologie marquaient un fort recul, les récriminations non dissimulées d'électeurs mécontents, les réunions publiques locales, la rupture avec Washington et la cyberdémocratie suivirent le même mouvement. Après la disparition de TalkBack Live le 7 mars 2003, il ne restait plus que Washington Journal, une matinale à antenne ouverte proposée par GSPAN et permettant la participation interactive des téléspectateurs ${ }^{32}$. Toutefois, la présente analyse met en lumière la volonté des producteurs du câble d'exploiter le contexte et l'humeur du moment, une tentative qui leur a fait, à leur tour, remettre en cause les normes en vigueur quant aux sujets politiques pouvant être débattus à la télévision et aux personnes habilitées à prendre la parole. America's Talking, National Empowerment Television, et TalkBack Live ont modifié le paysage audiovisuel en imposant l'idée qu'on ne pouvait pas se contenter de s'adresser au public, mais qu'on devait dialoguer avec lui. Ce dernier a donc pu prendre part aux discussions et se voir courtisé non seulement pour ce qu'il savait, mais également pour son discernement et son savoir-faire en matière de connexion aux réseaux et de partage des informations. Enfin, la télévision a démontré que la politique ne se réduisait pas au microcosme de l'administration washingtonienne, mais concernait également les gens ordinaires et leur vie de tous les jours. Selon leurs producteurs, ces chaînes et ces émissions ont permis aux citoyens de s'exprimer et de dialoguer avec des représentants du pouvoir et avec leurs compatriotes, ce qui équivaut à un véritable changement politique. À des degrés différents, ces trois programmes ont fait la promotion d'une certaine posture sur le thème du « nous contre eux », en attirant un public mécontent vers le discours politique.

\section{La transformation de l'information sur le câble}

L'héritage le plus marquant et le plus durable des programmes du type « voix du peuple » est sans doute l'étonnant succès de Roger Ailes, son influence et celle de ses héritiers sur l'évolution des émissions d'actualité sur le câble. Lorsque America's Talking devint MSNBC en 1996, Roger Ailes quitta la chaîne pour devenir directeur des programmes de Fox News. Alors que ses tentatives de tirer parti d'un discours ronflant, conservateur et populiste, avec le Rush Limbaugh Show et America's Talking avaient, dans l'ensemble, été infructueuses, il rencontra le succès en drapant ce

\footnotetext{
32 CNN a supprimé TalkBack en 2003, alors que l'Amérique s'apprêtait à entrer en guerre contre l'Irak. La chaine a précisé que cette décision était motivée par un contexte d'actualités intenses. Pourtant, alors que l'Amérique s'interrogeait sur l'utilité de cet engagement en Irak et que la plupart des alliés désapprouvait cette décision, CNN ne semblait pas intéressée par l'avis des téléspectateurs et du public sur le plateau. L'émission était suivie par 600 à 700000 téléspectateurs (Wilbert, 2003).
} 
même discours dans un manteau d'« objectivité » journalistique. En effet, tout en offrant un discours ouvertement conservateur et des reportages d'actualité empreints d'idéologie, la chaîne n'hésitait pas à se présenter comme « honnête et pondérée ». Elle persista dans son engagement « au côté des gens », réminiscence des antennes ouvertes au public de Roger Ailes avec, dans ses bandes annonces, des slogans du type « Les informations, c'est nous, les décisions, c'est vous ». D'une certaine manière, Fox News n'a pas cherché à nier l'étiquette conservatrice que les critiques lui avaient attribuée au prétexte que la chaîne estimait les Américains « persuadés que les médias d'actualités penchent à gauche et que, par conséquent, la chaîne est un moyen de contrebalancer cette tendance » (Trigoboff, 2002).

Depuis 1996, Fox News, CNN et MSNBC étaient les principales chaînes à proposer des émissions politiques sur le câble ${ }^{33}$ et dépendaient essentiellement des débats pour tenir financièrement en raison du fait que la couverture des informations sérieuses coûte cher. Si les chaînes traditionnelles avaient depuis toujours réussi à couvrir les dépenses des programmes d'actualité (ces derniers étaient si déficitaires que les services « information » des chaînes étaient qualifiés de « produits d'appel vendus à perte »), les chaînes d'actualité du câble n'ont jamais bénéficié de ce... luxe. Ainsi, dans un contexte de concurrence croissante entre les trois chaînes, ces dernières se sont-elles orientées vers le talk show qui constituait un moyen économique de remplir une grille de programme 24 heures sur 24 (Quenqua, 2002 ; Rutten, 2002). Pour finir, ces chaînes dites d'actualité ont fini par diffuser plus de talk shows que de véritables actualités.

Roger Ailes a déclenché une véritable offensive contre l'idée bien ancrée selon laquelle les actualités télévisées devaient se concentrer sur l'exposé des faits, de manière juste, pondérée et non partisane. Fox News a laissé libre cours à son ambition d'atteindre un très large public et s'est évertuée à promouvoir l'idéologie conservatrice en jouant des peurs du public et de sa volonté de vengeance après les attentats terroristes du II septembre 200 I. Dans le même temps, la chaîne est devenue la championne des mesures d'audience. L'humeur politique du pays avait changé : le public je-sais-tout vivant dans une Amérique sûre d'elle-même était maintenant composé de téléspectateurs avides d'explications à la suite d'un des événements les plus stupéfiants et traumatisants de l'histoire de leur pays ${ }^{34}$. En faisant apparaitre le drapeau américain à chaque coin de l'écran, Fox News s'appropriait l'emblème et sa revendication patriotique pour faire vibrer la corde émotionnelle des auditeurs, tout en œuvrant pour la cause de l'administration George W. Bush et

\footnotetext{
33 Même si, parfois, elle s'est aventurée sur le terrain du discours ouvertement politique (comme avec le show éphémère de Dennis Miller en 2004), la chaine CNBC s'est principalement concentrée sur des actualités et des débats sur le monde des affaires.

${ }^{34}$ D. Nimmo et J. E. Combs (1992 : 167-169) concluent que l'expertise présentée dans les talk shows de la télévision américaine subsiste car elle représente une sorte de « guérison symbolique » pour les téléspectateurs, une sorte de médecine curative faite de symboles et de mythes en ces temps troublés et complexes. La domination constante de la parole « d'expert » dans les débats du câble prouve le bien-fondé de cette remarque théorique.
} 
sa « guerre contre le terrorisme ». Dans cette optique, la chaîne reprit le leitmotiv du « nous contre eux », remontant à l'époque de « la voix du peuple », mais avec, cette fois, un ennemi différent. Bill O'Reilly fut choisi pour incarner la « voix du peuple », avec sa rhétorique du « sens commun » et sa façon bien à lui de dépasser les limites avec des invités qui s'en trouvaient déboussolés. Geraldo Rivera allait aussi incarner la « voix du peuple » : un soi-disant reporter envoyé par Fox News pour aller fouiller les grottes de Tora Bora en Afghanistan, un pistolet à la ceinture, et censé traquer Oussama Ben Laden, rien de moins! De fait, Fox News avait beau se présenter comme une chaîne " d'actualité », ses programmes bavards et au style propre (le discours des experts, les vociférations des animateurs ou les reporters globe-trotters) et son engagement aux côtés du téléspectateur pour mieux le séduire (que ce soit au moyen de stratagèmes comme les courriels, la présence du public sur le plateau, la couleur idéologique ou le zèle patriotique) ne faisaient que refléter un discours politique présenté sous un autre nom.

Dès lors que les ravages de l'administration George W. Bush commencèrent à se faire jour aux yeux des citoyens américains, redonnant ainsi une majorité au Parti démocrate à la Chambre des représentants à l'automne 2006, la cote de Fox News commença à suivre la baisse de popularité du président américain et de son viceprésident Dick Cheney, une chute qui allait se poursuivre tout au long des années de déclin de l'administration George W. Bush. Lors de l'élection présidentielle de 2008 notamment, les émissions de la Fox œuvrant bruyamment pour la cause républicaine étaient en total décalage avec l'engouement du public pour les deux principaux candidats à l'investiture démocrate (The New York Times, 2008). En 2007, mais surtout en 2008, MSNBC réussit à se forger une belle image de marque en programmant en prime time toute une série de talk shows présentés par des animateurs de gauche, comme par exemple Hardball with Chris Matthews, Countdown with Keith Olbermann et, plus tard, The Rachel Maddow Show ${ }^{35}$. À sa grande fierté, MSNBC devint alors la tribune privilégiée des téléspectateurs pour exprimer leur mécontentement et leur déception à l'égard de l'administration George W. Bush, ainsi que leur espoir de voir les Démocrates revenir aux affaires. II fut facile de prétendre, comme le firent un certain nombre de critiques, que MSNBC et Fox représentaient les deux faces d'une seule pièce, car elles proposaient chacune un discours idéologique destiné à séduire leurs publics respectifs en jouant sur la corde sensible des téléspectateurs. Cette évolution vers un manque total d'impartialité allait démontrer qu'aucune des deux chaînes n'était capable de « rendre compte » de la « réalité » politique avec précision et équité (Rutenberg, 2008).

La polémique suscitée par l'appartenance manifeste des deux chaînes à l'un ou l'autre camp politique - toutes deux affirmant que cela ne transparaissait que dans les débats en prime time et non dans les journaux télévisés - déborda jusque dans la campagne présidentielle, lorsque les Démocrates refusèrent à

35 MSNBC a engagé l'animateur P. Donahue, classé à gauche, au début des années 2000, mais a dû le remercier rapidement lorsque la ferveur patriotique s'est emparée du pays à la suite des attentats du I I septembre 200 I. 
Fox News d'accueillir un débat de leur parti en raison de sa partialité présumée (Montopoli, 2007). De même, lors de la Convention républicaine, les délégués et les responsables du parti s'en prirent violemment à NBC News, la maison mère de MSNBC, en déclarant qu'elle était contaminée par les idées de gauche qui régnaient en maître sur la chaîne câblée (Bauder, 2008). En conséquence, tandis que toutes les chaînes du câble bénéficiaient d'une campagne présidentielle qui suscitait un énorme intérêt du public (cNN tout particulièrement), se posait la question de savoir à quoi ressembleraient ces chaînes d'information engagées dans une concurrence idéologique acharnée, une fois la lutte partisane venue à son terme (Carter, 2008). À peu près à la même chose, semble-il.

Pendant l'ère Barack Obama, Fox News est remontée au sommet des taux d'audience (ces derniers ayant brièvement été à l'avantage de CNN durant la campagne présidentielle) en livrant une guerre idéologique retentissante contre Barack Obama et son administration quasiment dès son arrivée aux responsabilités en $2008^{36}$. La chaîne plaça la barre encore plus haut en engageant un animateur xénophobe d'un nouveau genre : Glenn Beck, qui - chose des plus étranges - appela à la rescousse Howard Beale, le personnage dérangé de Network (Lumet, 1976), lequel déclarait dans ce film : « Nous sommes furieux et il n'est plus question de supporter ça ». Glenn Beck faisait là référence à la menace qu'était supposée représenter cette nouvelle administration de « socialistes » et de «fascistes » pour la société et les valeurs américaines ${ }^{37}$. L'animateur fit rapidement grimper l'audience en concoctant très adroitement un mélange efficace de styles télévisuels familiers qui associait la véhémence de Bill O’Reilly, la sensibilité exacerbée du télévangéliste Jimmy Swaggart, la théâtralité de Geraldo Rivera et les techniques de vente de l'info-publicité pour faire passer un message d'apocalypse imminente aux accents hystériques. Le succès de Fox News était d'autant plus surprenant que Glenn Beck ne passait pas en prime time, contrairement aux présentateurs des talk shows des autres chaînes aux tendances politiques ouvertement affirmées, mais à l'horaire habituellement prévu pour les actualités de la journée, 17 heures (heure de la côte Est).

Sur MSNBC, la présentatrice - marquée à gauche - Rachel Maddow, ancienne journaliste radio sur Air America et première animatrice d'émission politique à revendiquer son homosexualité, attira un large public avec son talk show lancé en prime time au moment de l'élection de Barack Obama. Sous bien des aspects, Rachel Maddow est l'exact opposé de Glenn Beck. Titulaire d'un doctorat obtenu à Oxford, elle se disait mordue de politique et montra sans tarder qu'elle était capable de conduire toutes sortes de discussions politiques, notamment lors d'une interview avec le président des États-Unis Barack Obama durant laquelle ils abordèrent en détail la question de la rénovation du réseau électrique américain. Pour sa part, CNN avait plongé à la quatrième place des indices d'écoute en prime

\footnotetext{
${ }^{36}$ La meilleure analyse des attaques farouches de Fox News contre la nouvelle administration a sans doute été celle de Jon Stewart du Daily Show dans une rubrique intitulée «Tox News Fear Imbalance ».

${ }_{37}$ G. Beck accepte même la comparaison avec H. Beale : voir Brian Stelter et Bill Carter (2009).
} 
time (derrière sa propre chaîne d'information en continu), prouvant ainsi que sa stratégie modérée, efficace en période d'élection, l'était beaucoup moins au quotidien lorsque les auditeurs habituels du câble allumaient leur télévision le soir pour avoir un résumé des événements de la journée dans une optique partisane.

En résumé, les chaînes d'information du câble ont purement et simplement remis en question la vision classique des actualités télévisées. L'idéologie, voire la partialité, y jouent souvent les premiers rôles, mais les débats et les opinions, et non le travail journalistique, constituent leur véritable marque de fabrique. Au lieu de profiter de cette omniprésence sur les ondes (24 heures, contre 30 minutes pour les bulletins d'information des grandes chaînes traditionnelles) pour proposer des analyses pointues, les chaînes du câble ont préféré les débats partisans qui généraient beaucoup plus d'audience. Là encore, comme pendant l'ère de la « voix du peuple », les principaux messages politiques sont émis par des non-spécialistes : Gretchen Carlson, ex-miss America, présentatrice des informations du matin sur Fox \& Friends; Glenn Beck, Sean Hannity et Rachel Maddow, présentateurs de talk shows radiophoniques venus à la télévision ; Bill O'Reilly, ancien présentateur vedette d'émissions à sensation et Keith Olbermann, ancien présentateur sportif. Presque tous ont adopté ce style outrancier décrit par John Thorton Caldwell. De plus, alors que les grandes chaînes traditionnelles ne couvraient pour ainsi dire plus l'actualité politique ordinaire pour se concentrer sur les grands événements, notamment les débats présidentiels, les soirées électorales, et les discours sur l'état de l'Union, les chaînes d'information câblées sont devenues le lieu privilégié des actualités politiques. Finalement, c'est cette nouvelle conception des « actualités » qui est aujourd'hui adoptée par la grande majorité des émissions politiques quotidiennes de l'ère post-grandes chaînes nationales.

\section{Conclusion}

L'histoire du discours politique démontre que les frontières arbitraires entre experts politiques et non-experts sont aujourd'hui beaucoup plus floues qu'autrefois. Par ailleurs, les moyens mis en œuvre pour cibler avec précision et séduire le public de telle ou telle émission se sont très largement développés et diversifiés par rapport à ceux des précédentes générations d'émissions politiques. Toutefois, il reste à prouver que l'ambition des producteurs invitant « la voix du peuple » à se faire entendre au milieu des années 90 se soit réalisée, à savoir si elle a responsabilisé les téléspectateurs sur le plan politique en leur permettant de « dialoguer en direct » avec les dirigeants du pays. Quoi qu'il en soit, c'est par cette voie que les producteurs et les téléspectateurs ont pu redéfinir les critères de ce qui pouvait constituer un débat politique télévisé intéressant et attractif. Ainsi le spectacle d'un débat politique militant et hargneux s'est-il enraciné dans le succès de ces chaînes « d'information » métamorphosées. 


\section{Références}

Alterman E., 1999, Sound and Fury: The Making of the Punditocracy, New York, Cornell University Press.

Ball R., 1998, Meet the Press : Fifty Years of History in the Making, New York, McGraw-Hill.

Barker D. C., 2002, Rushed to Judgment : Talk Radio, Persuasion, and American Political Behavior, New York, Columbia University Press.

Bauder D., 2008, « Study : NBC News Doesn't Follow MSNBC's Partisan Drift », Huffington Post, 29 oct.

Brown R., 1994, « America's Talking Cable Channel Takes Off », Broadcasting Cable, I24, 4 juil., p. 16.

Buckley W. F. Jr., 1989, On the Firing Line, New York, Random House.

Caldwell J. T., 1995, Televisuality : Style, Crisis, and Authority in American Television, New Brunswick, Rutgers University Press.

Carpignano P., Anderson R., Aronowitz S., DiFazio W., 1993, « Chatter in the Age of Electronic Reproduction :Talk Television and the "Public Mind" », pp. 33-55, in : Robbins Br., ed., The Phantom Public Sphere, Minneapolis, University of Minnesota Press.

Carter B., 2008, « Election's Over, So What's Next for the Cable News Channels », The New York Times, I 5 nov., p. Cl.

Dallek R., 1999, Ronald Reagan :The Politics of Symbolism, Cambridge, Harvard University Press.

Dempsey J., 1994, « Newest Cable Act Child:America's Talking », Daily Variety, 27 juin, p. 32.

Dillin J., 1990, « American Voters Disgusted, Angry with Politicians », Christian Science Monitor, 17 oct., pp. 4-5.

Dionne E. Jr., 1991, Why Americans Hate Politics, New York, Simon and Schuster.

Dowd M., 1992, « Populist Media Forums and the Campaign of "92"», The New York Times, 3 nov., p. 14.

Gamson J., 1998, Freaks Talk Back : Tabloid Talk Shows and Sexual Nonconformity, Chicago, University of Chicago Press.

Gitlin T., 1995, The Twilight of Common Dreams: Why America Is Wracked by Culture Wars, New York, Metropolitan Books.

Glynn K., 2000, Tabloid Culture :Trash Taste, Popular Power, and the Transformation of American Television, Durham, Duke University Press.

Gore A., 1995, Common Sense Government: Works Better and Costs Less, New York, Random House.

Grindstaff L., 2002, The Money Shot :Trash, Class, and the Making of TV Talk Shows, Chicago, University of Chicago Press.

Hertzke A. D., 1993, Echoes of Discontent : Jesse Jackson, Pat Robertson, and the Resurgence of Populism, Washington, Congressional Quarterly Press.

Hirsch A., 1991, Talking Heads : Political Talk Shows and Their Star Pundits, New York, St. Martin's Press. 
Ivins M., 1996, Austin-American Statesman, 6 févr., p. A9.

Jones J. P., 2003, « Vox Populi as Cable Programming Strategy », Journal of Popular Film Television, 31, pp. 18-28.

King L., en collab. avec Stencel M., 1993, On the Line: The New Road to the White House, New York, Harcourt Brace and Company.

Klein J., 1995, «The Birth of Common Sense : Bill Clinton Outflanks the Republicans on Regulatory Reform », Newsweek, 125, 27 mars, p. 31.

Kloer P., 1994, « CNN Interactive Program Will Raise Back Talk to a New Level », Atlanta Journal and Constitution, I I juil., p. Al.

Kurtz H., 1996, Hot Air All Talk, All the Time, New York, Times Books.

Laufer P., 1995, Inside Talk Radio : America's Voice or Just Hot Air, New York, Carol Publishing Group.

Lipset S., SchneiderW., 1987, The Confidence Cap, Baltimore, Johns Hopkins University Press.

Livingstone S., Lunt P., 1994, Talk on Television : Audience Participation and Public Debate, London, Routledge.

Mansfield H., 1994, « Newt, Take note : Populism Poses its own dangers », Wall Street Journal, $\mid$ nov., A |

Marshall P. D., 1997, Celebrity and Power : Fame in Contemporary Culture, Minneapolis, University of Minnesota Press.

Montopoli Br., 2007, « Fox-Backed Democratic Debate Called Off », CBS News, 23 août.

Moss L., 1993, « C-SPAN with Attitude Will Launch December C », Multichannel News, I4, 15 nov., p. 14.

Mundy A., 1996, « Showtime in the Capitol », MediaWeek, janv., pp. 20-22.

Munson W., 1993, All Talk:The Talkshow in Media Culture, Philadelphia, Temple University Press.

Nimmo D., Combs J. E., 1992, The Political Pundits, New York, Praeger.

Oreskes M., 1990, « As Problems Fester,Voters Send Pink Slips », New York Times, 23 sept., pp. 4-5.

Patterson T., 1993, Out of Order, New York, Random House.

Pharr S., Putnam R., 2000, Disaffected Democracies : What's Troubling the Trilateral Countries?, Princeton, Princeton University Press.

Piccone P., Ulmen G., 1995, « Populism and the New Politics », Telos, 103, pp. 3-8.

Quenqua D., 2002, « MSBNC Shifts Focus from Reporting to Commentary », PR Week, 10 juin, p. 3.

Rheingold H., 1991, Virtual Community: Homesteading on the Electronic Frontier, New York, Simon and Schuster.

Rice M., 1994, « People Faxes, Computers Debate the Issues on New cnN Program », Associated Press, 22 août.

Rosak T., 1994, The Cult of Information, Berkeley, University of California Press.

Rutenberg J., 2008, «A Surge on One Channel, a Tight Race on Another », New York Times, I nov., p.A28. 
Rutten T., 2002, «Talk is Cheap, or at Least Cheaper Than Newscasts », Los Angeles Times, 7 juin, p. D2.

Schudson M., 1998, The Good Citizen :A History of American Civic Life, New York, The Free Press.

Schulte-Sasse L., 1993, « Meet Ross Perot: The Lasting Legacy of Capraesque Populism », Cultural Critique, 25, pp. 91-119.

Shapiro A., 1998, «Technorealism : Get Real ! », The Nation, 266, pp. 19-20.

Shattuc J. M., 1997, The Talking Cure : tv Talk Shows and Women, New York, Routledge.

Sokolsky B., 1994, « TalkBack Live Touts Town Meeting Format », The Press Enterprise (Riverside, CA), 5 août, p. B5.

Stelter B., Carter B., 2009, «Tox News's Mad, Apocalyptic, Tearful Rising Star », New York Times, 29 mars, p. Al.

The New York Times, 1995, « Bad Justice », 2 I févr., p. Al 8

— 2008, «Fox News Ratings Show Erosion », 28 juin.

Timberg B., 2002, Television Talk: A History of the tv Talk Show, Austin, University of Texas Press.

Tolson A., 1991, «Televised Chat and the Synthetic Personality », in : Scannell P., ed., Broadcast Talk, London, Sage.

Trigoboff D., 2002, « Nets : News, Views, Confused », Broadcasting \& Cable, I I mars, p. 10.

Westlind D., 1996, The Politics of Popular Identity, Lund, Lund University Press.

Wharton D., 1993, « Debuting Cable/Sat Net Tuned to the Right », Daily Variety, 29 nov., p. 4.

Wilbert C., 2003, « CNN Pulls Plug on Afternoon "TalkBack" », Atlanta Journal -Constitution, 8 mars, p. I E.

Wilentz S., 1995, « Populism Redux », Dissent 42, pp. I49-53.

Williams S., 1994, «America's Talking-The All-Talk Cable Network-Bows on July $4^{\text {th }}$ »,Associated Press, | $\left.\right|^{\text {er juil. }}$ 